\title{
Transforming the root of the problem
}

Here is a commercial biotechnology fantasy, if ever there was one: A dietary product that 500 million people not only need but already use. It is vital and indispensable, and yet it has many obvious deficiencies. One of this product's advantages, however, is that it is biological and, thanks to technology breakthroughs, it is now amenable to improvement through genetic engineering. Given all this, it is hard to believe that no company has seized the opportunity to exploit what surely must be an obvious niche.

Well, maybe it's not so hard-when we find out that the product is not a drug, nor a nutraceutical, nor a calorie-free nondairy creamerbut Manihot esculenta Crantz, the calorie-packed (but usually nonfattening) tropical delicacy, cassava. Three separate papers in this issue of Nature Biotechnology report the establishment of suspension cultures of cassava cells, their transformation, and subsequent regeneration.

There is little doubt that the transformation of this root crop staple is a remarkable technical feat. It is heartening, too, that Western organizations have had the foresight to devote precious government funds to this research. But it would be unrealistic to imagine that this advance is likely to have any significance very far beyond the purely technological.

The real difficulty for a product like cassava is that the machinery for grabbing hold of innovations and transforming them into useful products-if it exists at all--is unresponsive and unexplored. 'The path to the "market" is not merely stonier and less well trodden, it is a wilderness.

Research scientists and companies developing biological technology know already of the obstacles on the path that leads from the laboratory to the market. Very few of these hopeful research projects succeed. Most new chemical entities, for instance, fail to make it as drugs or agrochemical agents because they are too toxic, or ineffective, or unmanufacturable, or undeliverable. But at least the route to the market is familiar. There is, within all countries of the developed world, an infrastructure that facilitates the dissemination of innovation. That infrastructure is, simplistically speaking, cyclic. Fueled by capital, it proceeds through research, development, manufacturing, distribution, promotion, and retailing to consumers, and back again-through market research, the setting of priorities, and the allocation of funding--to research.

Little of this infrastructure operates for cassava. Optimism may allow one to assume that further research and development will be funded so that genes for disease resistances or stress tolerances or nutritional quality can be transformed into, maintained stably and expressed in, cassava cultivars. But that is not enough. Cassava has a "nebulous and dynamic gene pool"- as befits a crop that grows in tropical and subtropical regions. It grows at the margins, in poor soils, with thousands of local varieties, each adapted to local conditions. If cassava growers could apply fertilizers, weedkillers, or pesticides to provide a more comfortable environment, and thereby accommodate a narrower range of crop types, they would probably not grow cassava. Will there be enough research money to transform and then develop the thousands of different land-races of the crop that cassava "consumers" use?

Even if appropriate cultivars are developed, can they be "delivered" to farmers? Corn farmers and breeders have a communication network-roads, telephones, faxes, even electronic mail-that works. Despite this, convincing farmers to grow new varieties of corn is not easy-as any agricultural rep will relate. Cassava growers are vastly more numerous than corn farmers. They are remote from breeders, physically and socially. The two-way street of communication is seldom traveled.

Cassava is in danger of becoming the wrong kind of icon for biotechnology. It seems to represent a desire that the biology that has revealed global human kinship and genetic equality should serve, too, to balance practical inequities in human lives. For cassava, as for the rest of biotechnology, desire is only the start.

\section{Can ELSI's principles inform consensus?}

As the sequencing aspect of the Human Genome Project (HGP) slouches toward completion (sequencing may be completed as early as 2003, under budget and ahead of schedule), how will its products-like genetic testing-be incorporated into existing medical and research structures? One issue that brings some of the HGP's a-rose-with-bigthorns features to light is that of informed consent, particularly with respect to stored tissue research. Researchers worry that a headlong rush to ensure genetic privacy will cause informed consent procedures for genetic testing and research to become so convoluted that research gridlock will result. For example, if a researcher wants to use archived tissue samples being held (as they routinely are, at teaching hospitals and research institutes) to establish the frequency of a genetic mutation in a population of people who have already been screened for another condition in order to validate a genetic test, does the researcher need to get informed consent from these patients before doing so? And then for each subsequent new indication? And what about new sample collection? Patients and research participants, on the other hand, worry about the kinds of research their tissues, blood, DNA, and gametes are to be used for and who has access to the resulting information. Some feel they should be compensated for their material "contributions."

The Task Force on Genetic Testing of the NIH-DOE Working Group on the Ethical, Legal, and Social Implications (ELSI) of the HGP has drafted thoughtful interim principles addressing informed consent and a wealth of other issues as they pertain to genetic testing, and has invited public comment on the draft document.* Many of the recommendations concerning scientific validation of tests and laboratory quality assurance, as well as suggested measures for short circuiting discrimination, are commendable. Some will elicit howls of displeasure. Those with a stake in genetic research-researcher, physician, biotechnologist, genetic consumer-would be well advised to examine these preliminary recommendations, because they will provide the foundation for any US legislative or regulatory framework that eventually gets laid down in this area. And they should be a source of rich debate. Could a regulatory framework for genetic information be constructed around vigorous public discourse? Hard to imagine, but then so was sequencing some three billion base pairs just a short time ago.

*To request copies or make comments, contact Joshua Brown, Task Force on Genetic Testing, 550 N. Broadway, Suite 511, Baltimore MD 21205, 1-410-9557894 (p), 1-410-955-0241 (f), jbrown@welchlink.welch.jhu.edu,

http://infonet.welch.jhu.edu/policy/genetics 